Revue européenne des sciences sociales

European Journal of Social Sciences

52-1 | 2014

Politiques du libre accès en sciences humaines et sociales

\title{
Copyright et économie des revues académiques
}

Olivier Bomsel

\section{OpenEdition}

Journals

Édition électronique

URL : http://journals.openedition.org/ress/2673

DOI : $10.4000 /$ ress. 2673

ISSN : 1663-4446

Éditeur

Librairie Droz

Édition imprimée

Date de publication : 6 mai 2014

Pagination : 93-110

ISBN : 978-2-600-01829-6

ISSN : 0048-8046

Référence électronique

Olivier Bomsel, «Copyright et économie des revues académiques », Revue européenne des sciences sociales [En ligne], 52-1 | 2014, mis en ligne le 12 mai 2014, consulté le 20 avril 2019. URL : http:/l journals.openedition.org/ress/2673; DOI : 10.4000/ress.2673 


\title{
COPYRIGHT ET ÉCONOMIE DES REVUES ACADÉMIQUES
}

OLIVIER BOMSEL

MINES ParisTech - CERNA

olivier.bomsel@mines-paristech.fr

\begin{abstract}
Résumé. Les revues académiques servent à labelliser et contextualiser des résultats de recherche. La consultation des revues académiques en libre accès est un changement de modèle de l'exploitation de leurs marques éditoriales. Cet article examine l'utilité économique de ces marques et le modèle de plate-forme (marchés bifaces) de leur exploitation. Dans ce modèle, le copyright sert à faire payer des lecteurs fortement consentants. Néanmoins, si ceux-ci ne souhaitent plus payer pour accéder aux articles, les revues doivent faire payer les auteurs. Le consentement-à-payer des auteurs dépend alors de la valeur du service éditorial apporté par la revue. Les services de validation et de contextualisation offerts par les revues académiques semblent davantage menacés par la numérisation pour les sciences humaines et sociales que dans le domaine des sciences, techniques et médecine.
\end{abstract}

Mots-clés: copyright, économie numérique, libre accès, marques éditoriales, revues scientifiques, sciences humaines et sociales.

\begin{abstract}
Academic journals are used for selection, validation and contextualization of research results. Providing open access to these journals brings a change in their publishing exploitation model. This paper examines the economic utility of academic publishing brands and the platform model (two-sided market) of their exploitation. In this model, copyright is used for collecting the fees on the readers' side. Hence, if these do not want to pay for accessing the articles, the journals should charge the authors. Then, the authors' willingness-to-pay depends on the value of the publishing service provided by each journal. On the short term, the utility of Human and Social Sciences journals seems more challenged by the digitalization than the one of Science, Technology and Medicine journals.
\end{abstract}

Keywords: academic journals, copyright, digital economy, editorial brand, human and social sciences, open access. 


\section{I.UNE RÉVOLTE}

Depuis le milieu des années 1990, bibliothécaires, chercheurs et universités dénoncent l'emprise croissante des éditeurs de revues scientifiques sur l'ensemble des utilisateurs. Le mouvement pour le libre accès se structure dans les années 2000 (Pérès, 20II), jusqu’à entraîner, dans les années 20I0, des actions de boycott des éditeurs de la part de grandes universités du classement de Shanghai (Harvard, Berkeley, Oxford, UPMC...). Parallèlement, des pétitions sont lancées, la plus emblématique visant Reed-Elsevier, recueillant environ 15000 signatures à ce jour.

Aux États-Unis, le boycott a fait suite à un projet de loi de décembre 20 I v visant à interdire aux agences scientifiques d'imposer aux chercheurs financés par elles de publier en libre accès (Benjamin, 2012). Face à ce tollé, le gouvernement britannique et la Commission européenne ont lancé en 2012 des initiatives pour que les résultats des travaux financés sur fonds publics soient, dès 20I4, mis en accès gratuit au plus tard douze mois après publication. Selon le chancelier de l'Echiquier David Willetts et la commissaire européenne Neelie Kroes, ces décisions apporteront des avantages économiques «énormes» (Le Monde, I7 juillet 2012) et relèveront le retour sur investissement des 87 milliards d'euros dépensés annuellement en recherche et développement dans l'Union'.

Néanmoins, et en dépit des analyses de certains courtiers² ${ }^{2}$ les cours de bourse des éditeurs scientifiques, plombés par ces annonces en 2012, se sont nettement repris tout au long de 2013 (voir Figures I-3). Le spectre d'un accès gratuit généralisé en 20I4 n'effraie pas les investisseurs. Que faut-il alors penser de ces annonces? L'interdiction faite aux chercheurs de publier dans des revues payantes est-elle une menace crédible? Peut-on utilement se passer de revues scientifiques payantes? Les économies annoncées ont-elles un fondement? L'économie de l'édition académique repose-t-elle fatalement sur le copyright?

I Communiqué de la Commission européenne du 17 juillet 2012: <http://europa.eu/rapid/ press-release_IP-12-790_fr.htm>.

2 Voir les évaluations de Natixis sur Reed-Elsevier. 


\section{L'APPROCHE BASIQUE DU COPYRIGHT}

«Comment développer un nouveau modèle économique qui affranchira les revues scientifiques des spéculations mercantiles des éditeurs?» (Darnton, 20I2, p. I4). Depuis le début des années 2000, l'historien Robert Darnton, directeur de la bibliothèque d'Harvard, s'est fait le héraut de la dénonciation du système économique des revues scientifiques. En 2007, écrit-il,

l'abonnement à de nombreuses revues scientifiques excédait 20000 dollars par an. Nuclear Physics A \& B, par exemple, coûte 2I 000 dollars et les dépenses pour les périodiques dans les bibliothèques de recherche ont augmenté de $320 \%$ au cours des deux dernières décennies. Le prestige d'une publication dans les revues les plus chères est si important pour l'avancement professionnel, en particulier dans les sciences dures, qu'il est devenu impossible aux bibliothèques de convaincre les enseignants d'accepter la résiliation de certains abonnements. Elles doivent donc surmonter la pression exercée sur leur budget en sacrifiant les monographies au profit des périodiques (ibid., p. 168).

Ces arguments sont largement repris par l'ensemble de la communauté scientifique. La hausse spectaculaire des prix de certaines revues impacte les budgets des bibliothèques, et, dans une moindre mesure, ceux des universités. Par ailleurs, la réputation des laboratoires et la carrière des chercheurs est de plus en plus tributaire des publications académiques, ce qui engendre une demande très inélastique au prix. Faut-il pour autant chasser les marchands du temple en dénonçant «la spéculation mercantile des éditeurs»?

Pour Darnton, le problème se compare à celui de la numérisation des collections des bibliothèques par Google. Il faut éviter «la privatisation d'un savoir qui appartient à la sphère publique » en dépit du lobbying des ayants droit :

Numériser les collections et les vendre en ligne sans se soucier de garantir un libre accès à tous reviendrait à répéter l'erreur qui fut commise lorsque les éditeurs s'emparèrent du marché des revues scientifiques, mais à une échelle bien plus grande puisque cela ferait d'Internet un instrument de privatisation d'un savoir qui appartient à la sphère publique. Nulle main invisible ne viendrait alors corriger le déséquilibre entre intérêt général et intérêt privé. Seul le public peut faire cela, mais qui le représente? Certainement pas les législateurs qui ont adopté la «loi Mickey Mouse »3 (ibid., p. I 19). 
Au fond, cette formulation reste modérée. On a vu, en effet, avec la numérisation, surgir des points de vue autrement radicaux prônant l'abolition du copyright et son remplacement par des mécanismes de licence obligatoire (voir notamment Lessig, 2004). La requête de Darnton s'approche davantage de la problématique classique de la propriété intellectuelle, à savoir : l'information étant un bien public, comment équilibrer l'incitation à créer fournie par un droit exclusif avec l'intérêt général de la diffusion du message correspondant ? Généralement, le législateur répond à cette question par une limitation de la durée des droits, faisant ensuite passer l'œuvre ou la technologie protégée dans le domaine public. Mais, objecte Darnton, la durée du copyright ayant été portée à 70 ans après la mort de l'auteur - loi «Mickey Mouse »-, comment se protéger des abus de monopole associés à l'information scientifique?

Cette approche est reprise en chœur par les pétitionnaires qui jugent en outre que les incitations à créer sont fournies, non pas par les droits cédés aux éditeurs, mais par les crédits versés par les agences gouvernementales. Et donc qu'à ce titre, les éditeurs ne peuvent obtenir de droits exclusifs sur les œuvres dont les États sont, en quelque sorte, coproducteurs.

La controverse rebondit parmi les économistes. Dans un article paru en 20ı, Steven Shavell plaide pour l'abolition du copyright qui ne sert selon lui qu’à nourrir une profession d'intermédiaires («third party»), alors que la communauté scientifique abonde à la fois en offre et en demande le marché de l'édition. Dès lors, si le copyright était aboli, le contenu des revues serait fourni de manière concurrentielle tandis que leur prix de vente s'alignerait sur leur coût marginal, lequel, numérisation oblige, tend rapidement vers zéro. De là une diffusion maximale bénéfique tant aux auteurs qu’aux lecteurs, voire à la société toute entière. L’argument est contesté par Frank Müller-Langer et Richard Watt qui insistent sur le fait que ce qui importe aux auteurs n'est pas tant le nombre de lecteurs que la qualification du lectorat : «les quelques lecteurs d'une revue très prestigieuse s'avèrent bien plus utiles [valuable] que les nombreux lecteurs d'une 
publication médiocre» (Müller-Langer, Watt, 20ı, p. 49). La fonction économique de la revue est donc d’ajuster, de rapprocher l'utilité escomptée de l'auteur avec l'utilité potentielle des lecteurs. En cela, elle crée une valeur - qu’on dira éditoriale - en excès de la création/diffusion.

En d'autres termes, la seule création (et le copyright qui la protège) ne suffit pas à justifier l'utilité ou la valeur des œuvres publiées. Pour la science, comme pour beaucoup d'autres domaines sujets à médiatisation, la valeur d'un bien, son utilité sociale, n’est pas tant l'expression consignée, que le protocole éditorial par lequel elle est rendue publique. Expliquons-nous.

\section{PROTOCOLES ÉDITORIAUX}

Une expression quelle qu'elle soit porte une signification différente selon le contexte dans lequel elle apparaît. Rien ne distingue a priori un fait divers d'une fiction. C'est la publication par un journal qui donne au fait divers son statut, et par là, sa signification. Selon que le journal est local ou national, la portée du fait divers sera très différente. Il en va de même pour le résultat d'une recherche scientifique. Rien ne distingue a priori un résultat juste d'une approximation, un récit d'une expérience réelle, une problématique cohérente d'un jeu d’hypothèses floues. La validation et la mise en contexte d'un résultat scientifique - comme pour la presse celle d'un fait social - fait partie du protocole éditorial ${ }^{4}$.

Dans l'édition scientifique - comme d'ailleurs dans le journalisme - cette validation prend deux dimensions : la revue par des pairs engageant leur crédit dans l'évaluation d'un travail-le comité de rédaction -, la responsabilité de l'éditeur estimant l'utilité de la recherche en regard de l'intérêt de ses lecteurs. La revue par des pairs est une caution scientifique, l'acceptation par la revue, un choix de contextualisation.

C’est précisément parce que la publication est un test d'utilité fondamental que la recherche est évaluée selon cet indicateur. En prônant la suppression, ou le contournement de ce test, c'est l'ensemble du système de production scientifique qu'on déstabilise. Le fait que certaines revues se vendent très au-dessus

4 Pour une élaboration du concept, voir Bomsel (dir.), 2013b. 
de leurs coûts de fabrication signifie certes qu'elles sont en concurrence imparfaite, mais aussi que la recherche qui s'y publie est extrêmement utile. Si elle ne l'était pas, les acheteurs n'en paieraient pas le prix. Il est paradoxal d'entendre des financiers de la recherche soutenir qu'il faut attendre des économies «énormes» de la suppression de leur premier critère d'évaluation.

$\mathrm{Si}$, pour des raisons qu'on va voir, le protocole éditorial de la science s’avère parfois trop coûteux, ce n'est pas en prônant sa suppression qu'on relèvera l'efficacité économique de la recherche. Au contraire, on détruira de la valeur dans la sélection, la validation et la contextualisation des résultats. Et, dans la concurrence internationale, cette destruction affectera d'abord les États ayant unilatéralement renoncé à l'édition payante 5 . Le caractère irréaliste de cette démarche explique, nous semble-t-il, la bonne tenue en bourse des éditeurs.

Un protocole éditorial procède par adjonction de marques qui signalent l'utilité d'un produit. Ces marques éditoriales, qu'il s'agisse des noms d'auteur, de laboratoire, d'université, de revue ou d'éditeur, concourent à la contextualisation des travaux. Chacune d'entre elles ajoute du sens, de l'utilité, de la valeur à l'expression publiée. Dans cette affaire, le droit exclusif d’accès au talent ou à l'expression (le copyright) est une garantie de la valorisation des marques éditoriales. Les universités paient les chercheurs comme les studios paient des scénaristes. Elles attendent en retour des gages d'exclusivité afin que leurs marques ne soient pas parasitées.

Il est légitime que les revues qui engagent leurs marques éditoriales puissent, elles aussi, bénéficier de l'exclusivité des travaux publiés. Le copyright est le droit cadastral sur lequel se construisent les marques des auteurs, des laboratoires, des universités, des revues, des éditeurs, etc. (Bomsel, 20Iza). Si les universités en pointe dans le boycott des éditeurs sont parmi les mieux classées du monde, ce n'est pas parce que les dépenses de documentation grèvent lourdement leurs budgets. On parle ici de millions d'euros face à des milliards

5 Si tous les pays ne passent pas simultanément au libre accès, les premiers à renoncer à l'édition payante perdront des équipes et des projets de recherche. Ce point est évoqué par Adam Tickell, vice-chancelier de l'université de Birmingham, interrogé par The Guardian et cité par Le Monde (17 juillet 2012): «Dans deux ans, la recherche publique britannique sera sur le Web». 
de frais de fonctionnement. C'est parce que leurs marques éditoriales - leur caution scientifique - sont à ce point puissantes qu'elles ont intérêt à affaiblir les grands éditeurs. Les grandes universités anglo-saxonnes ont déjà leurs maisons d'édition d'ouvrages savants. Elles pourraient les étendre à de nouvelles formes de médiatisation de travaux scientifiques.

Le problème soulevé par le relèvement du prix des revues scientifiques n'est donc pas lié directement à l'existence du copyright, ni même à sa durée, mais à l'économie des marques éditoriales qui s'y superposent. Or, cette économie est source de monopoles et de rentes. C'est cet aspect qui mérite analyse et pour lequel, en première approche, des remèdes doivent être recherchés.

\section{4. ÉCONOMIE DES MARQUES ÉDITORIALES}

Une marque éditoriale est un marché à deux versants. Elle s'adresse à la fois à des auteurs auxquels elle délivre un contexte et une «autorité», et à des lecteurs auxquels elle signale des travaux à forte utilité. Les marchés à deux versants bénéficient d'effets de réseau (Tirole, Rochet, 2003), autrement dit, ils sont d'autant plus utiles à chacun des groupes d'utilisateurs que ceuxci sont nombreux à les utiliser. Plus une marque attire d'auteurs de qualité, plus est forte l'utilité qu'elle délivre à ses lecteurs, et réciproquement. Si la qualité obéit à des critères objectifs - de validité ou de portée des résultats, par exemple -, de telles plate-formes ont vocation à devenir des monopoles sur chaque marché où elles opèrent. C'est le cas des revues de sciences techniques et médicales (STM), dont, en raison de la spécialisation des chercheurs, les marchés sont ultra-segmentés. Plus interdisciplinaires, moins objectivables, les sciences humaines et sociales (SHS) s'éditent également à travers des ouvrages : le pouvoir de marché des marques éditoriales y est donc moins flagrant ${ }^{6}$.

6 Certaines revues d'économie n'en demeurent pas moins très prestigieuses: American Economic Review, Econometrica, Quarterly Journal of Economics, Journal of Political Economy, Review of Economic Studies, International Economic Review, Journal of Economic Theory, Review of Economics and Statistics, etc. 
La règle tarifaire des plate-formes monopolistes (dit Ramsey-Boiteux) ${ }^{7}$ consiste à faire payer davantage les consommateurs les plus inélastiques au prix. La presse gratuite, par exemple, offre ses articles aux lecteurs et fait payer les annonceurs pour les encarts publicitaires. Elle touche ainsi un lectorat plus vaste dont elle fait payer l'accès davantage aux annonceurs. La demande des chercheurs pour les revues scientifiques étant fortement inélastique au prix, les éditeurs ont intérêt à les faire payer davantage que les auteurs.

Trois facteurs expliquent cependant que les prix puissent flamber au-dessus de l'optimum :

a) la très forte inélasticité de la demande face au monopole des marques attirant les meilleurs auteurs ${ }^{8}$;

b) la concentration de marques complémentaires, élargissant le monopole, au sein de firmes mondialisées ${ }^{9}$;

c) la vente groupée de ces marques sous forme de bouquets, selon des modèles d’abonnements à des bases de données numérisées.

Concentration et numérisation ont permis d'étendre le pouvoir de monopole de quelques revues puissantes à l'ensemble des catalogues des grands éditeurs, lesquels en ont profité pour relever leurs prix. Il est à noter qu'entre 2000 et 2012 , les cours de bourse de Reed Elsevier (Figure I) ou de Wolters Kluwer (Figure 2) ont moins bien performé que les autres sociétés de la cote, ce qui tend à prouver que les hausses de tarifs ont servi à financer les investissements consentis pour la numérisation dans toutes les activités d'édition de ces groupes ${ }^{10}$.

7 Le tarif de Ramsey-Boiteux est une règle de politique économique montrant que le prix qu'un monopole doit fixer pour maximiser le bien-être social se situe au-dessus de ses coûts. La règle indique que les services doivent être tarifés au-dessus du coût en proportion de l'inélasticité de la demande au prix pour ces services.

88000 revues font l'objet d'un classement bibliométrique par l'ISI, lequel sert de référence aux auteurs ainsi qu'aux administrateurs de la recherche.

9 «Elsevier-Sciences (hollandais, 2494 titres dont 253 en sciences sociales), Springer-Kluwer (suédois, environ 2000 titres dont 206 en sciences sociales), Wiley-Blackwell (anglais, 1492 titres dont 500 en sciences sociales), et Taylor \& Francis (anglais, 1300 titres dont 543 en sciences sociales). À titre de comparaison, le plus important éditeur français de STM, EDP Sciences, dispose de 50 titres. 》 (Pérès, 20II).

I0 Plus spécialisé, Wiley-Blackwell (John Wiley \& Sons), surperforme la cote dès le début des années 2000 (voir Figure 3). 
Voyant que les prix croissaient plus vite que l'utilité, les clients lecteurs se sont légitimement alarmés et ont entrepris, à leur tour, de se concentrer ${ }^{11}$. Mais le caractère insubstituable de l'offre ne leur a pas permis d'échapper aux hausses de prix. Face à cette impuissance, les chercheurs, encouragés par de grandes universités, ont mis en avant la thématique de l'accès libre, seule capable d'engendrer des offres de substitution. Et, malgré la position publique américaine, un influencée par le lobby d’Hollywood ${ }^{12}$, les politiques britanniques et européens ont suivi.

Or, l'inconvénient de cette approche est qu'elle ne vise pas la pratique commerciale ou la domination abusive des grands éditeurs en tant que telle, mais impose à tous les éditeurs, et surtout aux dominés, des règles altérant leur modèle économique. Elle prive alors la recherche, notamment dans les SHS, de moyens d'édition et de médiatisation.

\section{UN CASSE-TÊTE ÉCONOMIQUE}

Dans une activité plus explicitement commerciale que la recherche, il est possible que les grands comptes aient assigné les éditeurs pour pratique anticoncurrentielle ou abus de position dominante. L'idéologie du bien public, la tradition libertaire des chercheurs et le mouvement anti-copyright agité par les firmes du numérique ont masqué la dimension micro-économique du problème, la rejetant alors vers la sphère politique.

1 «Les consortiums de bibliothèques existent depuis les années 1930 aux États-Unis mais ils ont connu récemment, à la fin des années 1990, un nouveau développement [...]. En France, le consortium Couperin, fondé en 1999, associe 200 membres (Universités, Grandes Écoles, centres de recherche).» (Pérès, 201I).

12 Le Congrès, le Sénat, et le Président Obama ont successivement validé, en janvier 2014, le Consolidated Appropriation Act 2014 (HR 3547), qui entérine l'Open Access (OA) dans la loi. Pour mémoire, les États-Unis avaient progressivement imposé l'OA: en 2008 pour le NIH (recherche médicale) uniquement, puis début 2013 dans une directive de la Maison Blanche. Cette fois, l'OA fait force de loi, indépendamment de la volonté d'un directeur d'agence ou d'un parti politique au pouvoir. Ce texte s'impose aux agences fédérales financés (en partie) par le gouvernement, et qui dépensent plus de $100 \mathrm{M} \$$ en R\&D par an. II les oblige à rendre disponible gratuitement, au bout d'un an au plus, leurs publications. 
Cependant, les outils donnés par le droit de la concurrence sont d'un maniement subtil. En particulier, la démonstration de l'abus de position dominante est techniquement et juridiquement difficile car ce n'est pas le monopole qui est sanctionné, mais son abus, c'est-à-dire l'éviction d'éventuels concurrents. Or, dans ce cas précis, il n’y a pas d'éviction car les revues prestigieuses ne sont en monopole que sur leur champ de compétence. En outre, sur les marchés à deux versants, la démonstration d'éventuels comportements prédateurs se complique de ce que les prix élevés d'un monopole sur une des faces (les universités), peuvent être légitimement reversés en subvention aux utilisateurs (les auteurs) sur la seconde (voir Daley, Stryswoska, 20ı2).

Les abus associés aux clauses de ventes liées, auxquelles correspondent les abonnements proposés par les éditeurs scientifiques, sont souvent plus faciles à démontrer. Mais, là encore, il faut prouver qu'ils nuisent à la concurrence. Autrement dit, encore faut-il démontrer qu'un éditeur qui vend sa base de données comme produit lié à des revues en monopole, fait du tort à un autre qui ne peut alors vendre la sienne ou certains de ses produits. Or, tous les éditeurs ayant des revues en monopole, les tarifs pratiqués par les uns ne nuisent pas a priori aux autres. Il n’y a pas, à ce jour, d'éditeur ayant assigné un collègue pour forclusion ou concurrence déloyale.

En matière de prix, l’abus n'est caractérisé que si le bien ou service tarifé a statut de «facilité essentielle», auquel cas le tarif doit être orienté vers le coût de cette facilité. Selon le glossaire des termes employés dans le domaine de la politique de concurrence de l'Union européenne, une facilité essentielle désigne «une installation ou infrastructure nécessaire pour atteindre les clients et/ou pour permettre aux concurrents d'exercer leurs activités. Une facilité est essentielle lorsque sa reproduction est impossible ou extrêmement difficile en raison de contraintes physiques, géographiques, juridiques ou économiques» (Office des publications officielles des Communautés européennes, 2002).

L’exemple classique est celui d’un réseau électrique national utilisé par les différents producteurs d'électricité pour approvisionner les consommateurs finals : dans la mesure où il ne serait pas viable pour ces producteurs de construire leur propre réseau de distribution, ils dépendent de l'accès à l'infrastructure exis- 
tante. Le fait de refuser l'accès à une facilité essentielle peut être considéré comme un abus de position dominante de la part de l'entité contrôlant cette facilité, notamment lorsque cela empêche toute concurrence sur un marché situé en aval.

Dans le cas des revues scientifiques, les universités pourraient faire valoir que certaines d'entre elles sont des facilités essentielles dès lors qu'elles sont insubstituables et nécessaires à la conduite des recherches de leurs équipes. Celles-ci fournissent un service aval et complémentaire à celui des revues. Mais, là encore, il faudrait montrer que la concurrence entre laboratoires s'en trouve altérée, ce qui, compte tenu du financement mixte de la recherche, n’a, a priori, rien d'évident.

Les règles usuelles du droit de la concurrence semblent peu adaptées pour réguler efficacement les excès tarifaires reprochés à certains éditeurs. En outre, les accords de licence entre éditeurs et universités ressortissent à des contrats privés protégés par le secret commercial. Le marché est donc, par essence, très opaque, ce qui profite aux acteurs puissants. Il est légitime alors de rechercher d'autres approches pour tenter de le réguler.

\section{QUE CHANGE LE LIBRE ACCÈS?}

\section{I.POUR LES MARQUES EN MONOPOLE}

Au plan institutionnel, il abolit la fonction du copyright sans pour autant détruire celle des marques éditoriales. Les revues conservent leurs fonctions éditoriales - labellisation et contextualisation des travaux - mais doivent les monétiser différemment.

D’un point de vue économique, cela signifie que l'élasticité de la demande au prix des revues va tendanciellement augmenter. Les lecteurs voudront de moins en moins payer pour consulter les revues. La progressivité est importante car on imagine mal les universités boycotter dès 2014 des revues insubstituables dans les STM. Pour que les prix baissent et que les effets des ventes liées s'estompent, il faut attendre que les revues prestigieuses deviennent plus substituables, c'est-à-dire que leur contenu payant soit sensiblement réduit. En attendant, elles évoluent vers un modèle hybride et acceptent, si besoin, une mise en accès gratuit des contributions qui leur imposent cette contrainte, tout 
en imputant les frais de publication à leur(s) auteur(s) selon les modalités de la voie dorée (Gold Open Access). Néanmoins, une étude récente de Müller-Langer et Watt (20I3) montre que les chercheurs sont peu incités à financer des articles en libre accès dans des revues hybrides car un tel accès gratuit n’accroît pas mécaniquement le nombre de citations. Le surcoût demandé aux auteurs pour le libre accès à leurs articles n’a pas de contrepartie suffisante dans la diffusion. Le modèle ne commencera donc à basculer que lorsque les contraintes administratives sur la publication des travaux financés par des agences américaines et européennes auront un impact tangible. Ceci explique, selon nous, la bonne tenue des cours des grands éditeurs en 2013.

Cela dit, l'élasticité accrue de la demande universitaire aux prix incite les éditeurs à faire payer l'autre versant, c'est-à-dire les auteurs. Dès lors, soit l'utilité de la publication labellisée augmente et les éditeurs en position dominante vont tarifer les auteurs de plus en plus cher ${ }^{13}$, soit cette utilité n'augmente pas, ce qui veut dire que le pouvoir de labellisation et de contextualisation des revues diminue. C'est là que s'introduit la discussion sur les sciences humaines et sociales (SHS).

En effet, dans les domaines pour lesquels la validation et la contextualisation des revues ont un impact décisif sur l'accès des laboratoires aux financements publics et privés, l'utilité de ces revues ne sera pas remise en cause. Les laboratoires consentiront à payer des prix élevés pour la soumission et la publication des articles. Le coût de la validation et de la médiatisation de la recherche sera financé par le laboratoire de la même manière qu'une entreprise finance une homologation ou une mise en marché. Au gré de la contrainte sur l'accès libre, les revues migreront progressivement vers un modèle financé davantage par les laboratoires et moins par les services centraux des universités ${ }^{14}$. Les agences gouvernementales continue-

13 King et Alvarado-Albertorio (2008) recensent des prix compris entre I 250 et 3000 US\$ pour une publication en accès libre dans une revue de bon standard. Ce prix peut intégrer une composante à fonds perdu pour la soumission de l'article. Dans ces conditions, la publication d'une dizaine d'articles par un laboratoire équivaut au montant de l'abonnement universitaire annuel à une revue de prestige en accès payant.

14 Ce système engendre néanmoins des coûts de contrôle des droits des articles en libre accès. Les éditeurs académiques, comme les autres ayants droit de la propriété intellectuelle, doivent notifier les plates-formes et les auteurs des mises en accès abusifs d'articles sous copyright. 
ront de payer pour les revues, mais davantage à travers les coûts de soumission des articles. Selon Müller-Langer et Watt (2010, p. 55), les laboratoires qui publient le plus dépenseront davantage qu'ils n'économiseront en frais d'abonnement. Ils attireront ainsi les meilleurs chercheurs de leur discipline. À l'inverse, les universités les moins cotées feront des économies, mais auront du mal à recruter. Des systèmes de mutualisation entre laboratoires ou universités peuvent être imaginés, mais on voit mal les universités les plus cotées subventionner les universités moins prestigieuses. Au total, il n'est pas certain, surtout si les taux de rejet sont élevés et que les revues conservent des articles en accès payant, que le nouveau modèle engendre des économies.

En outre, tant que les revues conserveront un pouvoir de monopole sur la labellisation, les prix demandés aux auteurs pourront se maintenir durablement bien au-dessus du coût de validation. L'édition ne peut devenir un bien public tant que certaines marques éditoriales demeurent privées. Une concurrence entre marques publiques et privées peut durablement exister pour la labellisation. Le scandale français du Mediator montre la faillibilité des procédures publiques de labellisation des médicaments... Il en va de même pour toute la science. En outre, l'État serait à la fois juge et partie. Un laboratoire paiera davantage pour être labellisé par une marque prestigieuse et indépendante s'il peut s'en prévaloir auprès de ses sponsors. La tarification gratuite ou le libre accès des articles aux lecteurs ne change pas grand chose au problème. L'économie des marques éditoriales permet de faire payer sans limite une labellisation, voire un cautionnement, de grand prestige. Un éditorial dans Le Monde n’a pas le même statut que dans l'Écho d'Enghien-Montmorency. La question est de savoir combien de temps ces marques demeureront différenciantes pour les lecteurs.

\subsection{POUR LES MARQUES EN CONCURRENCE}

Dans le domaine des SHS, on l’a dit, le pouvoir de marché des marques éditoriales est a priori plus fragile. Ceci veut dire qu'à l'exception de quelques grandes revues - une quinzaine en économie, quelques unités dans des disciplines moins courues - la validation et la contextualisation sont plus aisément substituables. En témoigne l'essor important de revues en libre accès «doré»(Gold OA), 
i.e. offrant, contre paiement par les auteurs, une validation par des pairs et une mise en ligne rapide des articles ${ }^{15}$. Ces revues sélectionnent et démarchent directement les auteurs sur la base de leurs publications antérieures. Bien entendu, le dynamisme commercial des éditeurs, non sanctionnés par les abonnements de lecteurs, prête à des dérives sacrifiant la sélection. Cela n’aura qu’un temps. Il est probable quau fur et à mesure, les scandales issus de l'incurie des réviseurs aidant $^{16}$, de nouveaux critères de qualité (parrainage, comité éditorial, taux de rejets, facteur d'impact, etc.) émergent et trouvent à se monétiser.

Quoi qu'il en soit, la transdisciplinarité, le caractère plus ou moins appliqué des travaux, l'extension potentielle du champ des lecteurs, favorisent la multiplicité des titres. L’usage systématique des mots-clés, des codes disciplinaires et des moteurs de recherche encourage l'exploration thématique indépendamment de la contextualisation par les revues. Le point critique est qu'en dehors des quelques revues de référence, c'est le titre des articles et la densité des liens HTML pointant vers eux (le nombre de citations) qui les fait surgir en tête des résultats de requêtes. Les plates-formes et logiciels spécialisés de recherche et d'aide à la citation d'articles renforcent cette tendance ${ }^{17}$. La contextualisation est alors donnée par le nom des auteurs, la liste de leurs travaux, de leurs affiliations, la «marque » de leurs universités ou laboratoires, toutes informations accessibles en ligne et qui, peu à peu, prennent le pas sur le titre des revues. En termes économiques, les marques que portent toutes les instances de production scientifique sont engagées dans de nouvelles formes de concurrence intégrant le protocole éditorial. La numérisation qui supprime la barrière des coûts logistiques de la diffusion, mais renforce le pouvoir des marques éditoriales, intensifie la concurrence des éditeurs avec leurs fournisseurs et leurs clients.

15 Selon Pérès (201I), le DOAJ (Directory of Open Access Journal) en recensait 6754 en 201 I. En janvier 2014, il recense 9804 revues et I 573847 articles individuels.

16 Voir notamment le scandale Stapel découvert en août 20II (Barthélémy, 2012).

17 On pense à SSRN (Social Sciences Research Network) ou à la plate-forme Mendeley rachetée et opérée par Elsevier. 
La question qui se pose aux éditeurs de revues de sciences sociales est de s'adapter à ce nouvel environnement. Paradoxalement, ce qui est en jeu n'est pas la conservation ou non du copyright, mais la pérennisation de la valeur éditoriale de leurs marques. Autrement dit la valeur sémantique, le pouvoir contextuel, l'adjonction de sens que ces revues, ces marques éditoriales, offrent aux articles qu'elles rassemblent. De cette adjonction se déduit le consentement à payer des auteurs pour y publier, éventuellement en accès libre, et celui des institutions pour s'y abonner. Quel que soit le modèle retenu pour valoriser la marque - accès libre doré ou abonnement payant - le problème des revues de SHS est de s'adapter à un environnement dans lequel, contrairement aux revues de sciences et techniques, les fonctions traditionnelles de l'édition scientifique - validation, contextualisation - peuvent être dégroupées et offertes séparément.

Or, les critères de validation des SHS étant souvent moins formels que ceux des STM, cette fonction est aisément substituable et se prête davantage à la concurrence. Si, en outre, le contexte d'une revue est banal, sa marque est indifférente et les auteurs paieront la labellisation à son coût marginal. Sinon, l'éditeur peut espérer davantage... Le problème des SHS est que la labellisation y est plus floue que dans les STM et la contextualisation plus attaquée par les moteurs de recherche.

Dès lors, il nous semble que l'organisation thématique proposée par les revues de SHS joue un rôle critique dans la valorisation des articles qu'elles rassemblent. Le développement de numéros, voire de collections thématiques rassemblant des contributeurs identifiés et parrainés, représentatifs d'une approche transdisciplinaire, pourrait ainsi contribuer à créer de nouveaux contextes mieux résistants à la concurrence des moteurs de recherche et des labels universitaires. C'est, nous semble-t-il, tout le succès qu'il faut souhaiter à ce numéro. 
Figure I : Reed Elsevier PLC (RUK.N) VS NYSE composite (Reuters)

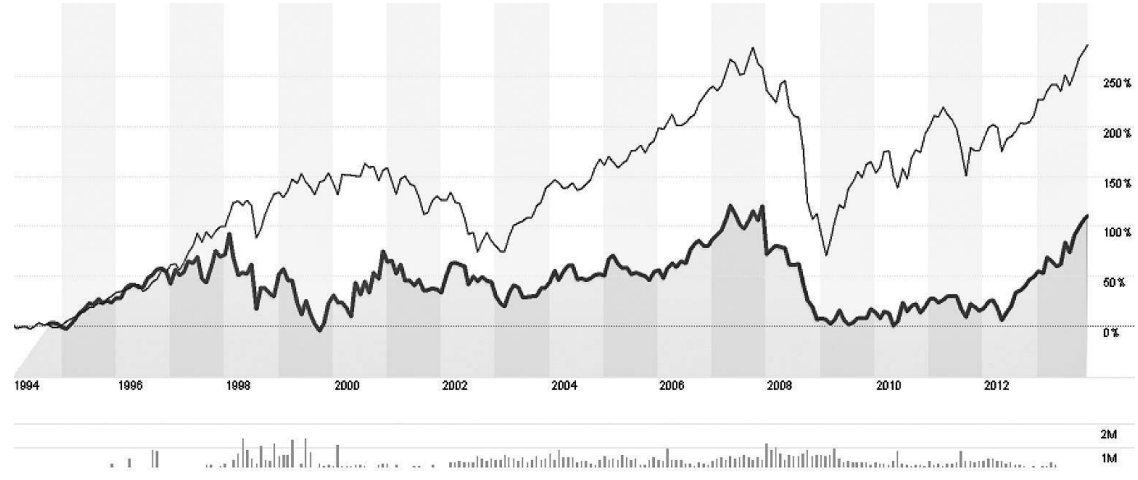

Figure 2: Wolters Kluwer NV (WLSNc.AS) VS NYSE composite (Reuters)

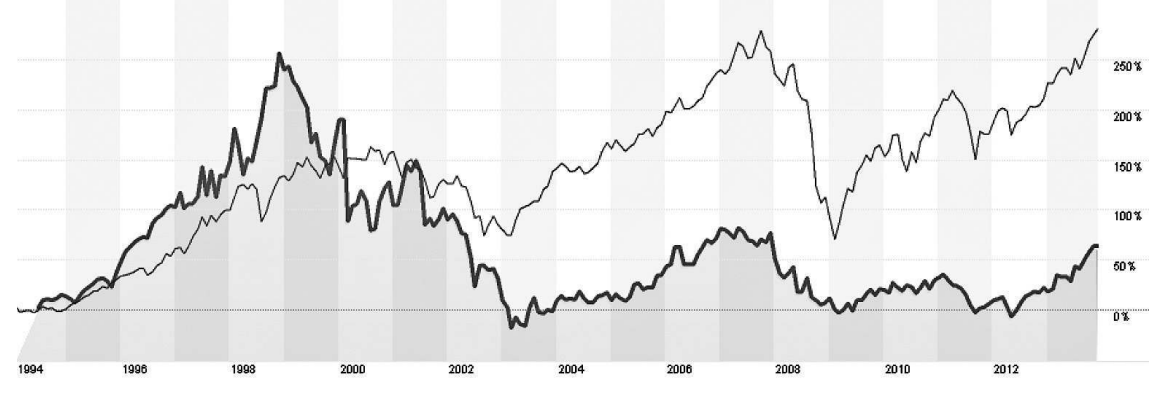

$100 \mathrm{M}$

Figure 3 : John Wiley \& Sons Inc (JWa) VS NYSE composite (Reuters)

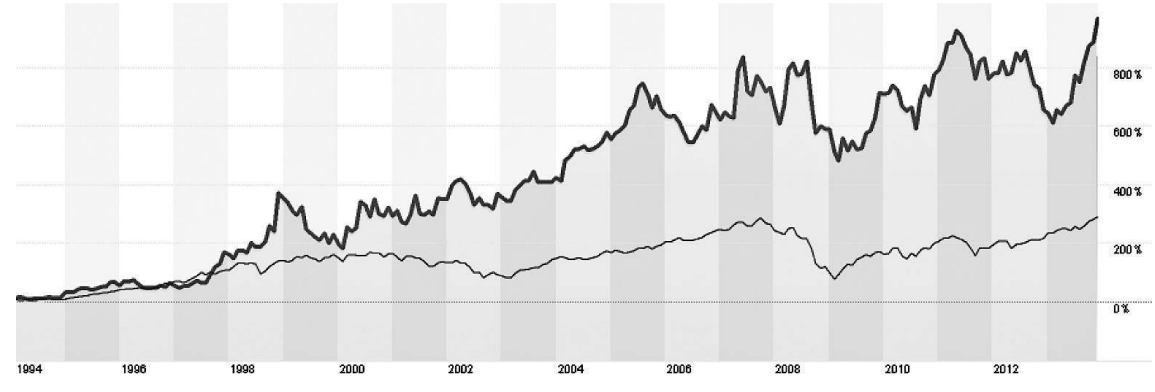

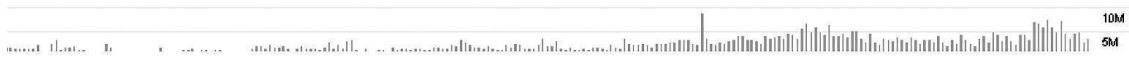




\section{BIBLIOGRAPHIE}

BARTHÉLÉMY Michel, 2012, «Le scandale Stapel, ou comment un homme seul a dupé le système scientifique», Blog Passeur de Sciences, 9 décembre: $<$ http://passeurdesciences.blog.lemonde.fr/20 I2/I2/09/le-scandale-stapel-oucomment-un-homme-seul-a-dupe-le-systeme-scientifique>.

BENJAMIN Anna, 20I2, «Harvard rejoint les universitaires pour un boycott des éditeurs», Le Monde, 25 avril: <http://lemonde.fr/sciences/ article/20 12/04/25/harvard-rejoint-les-universitaires-pour-un-boycott-des-editeurs_|69||25_|650684.html>.

BOMSEL Olivier, 2013a, «Copyright and Brands in the Digital Age», Contemporary Economic Policy, 31-I, p. I26-134.

-, (dir.), 2013b, Protocoles éditoriaux - Qu'est-ce que publier?, Paris, Armand Colin.

DALEY Nathalie, STRYSWOSKA Marta, 2012, «Spécificités des marchés bifaces: davantage de prudence dans l'évaluation des effets unilatéraux d'une fusion?» Le Cercle des Échos, 22 juin.

DARNTON Robert, 2012, Apologie du livre, Gallimard, «NRF Essais».

KING Donald W., ALVARADO-ALBERTORIO Frances M., 2008, «Pricing and Other Means of Charging Scholarly Journals: A Literature Review and Commentary», Learned Publishing, 21-4, p. 248-72.

LAWRENCE Lessig, 2004, Free Culture. How Big Media Uses Technology and the Law to Lock Down Culture and Control Creativity, New York, The Penguin Press.

Le Monde, 2012, «Dans deux ans, la recherche publique britannique sera sur le Web », 17 juillet: <http://lemonde.fr/education/article/2012/07/17/dans-deux-ansla-recherche-publique-britannique-sera-sur-le-web_|734400_|473685.html>.

MÜLLER-LANGER Frank, WATT Richard, 2010, «Copyright and Open Access for Academic Works», Review of Economic Research on Copyright Issues, 7-I, p.45-65.

-, 2013, «The Hybrid Open Access Citation Advantage: How Many More Cites is a $\$ 3000$ Fee Buying You?», 2013, Society of Economic Research on Copyright Issues Annual Conference (SERCl). 
OFFICE DES PUBLICATIONS OFFICIELLES DES COMMUNAUTÉS EUROPÉENNES, 2002, Glossaire des termes employés dans le domaine de la politique de concurrence de l'Union européenne, Direction générale de la concurrence, Bruxelles. PÉRÈS Jean, 20II, «L'édition scientifique», ACRIMED: <http://www.acrimed.org/ IMG/pdf/232L_edition_scientifique_I_et_2_.pdf>.

ROCHET Jean-Charles, TIROLE Jean, 2003, «Platform Competition and Two-Sided Markets», Journal of the European Economic Association, I-4, p. 990- 1029.

SHAVELL Steven, 2010, «Should Copyright of Academic Works be Abolished?» The Journal of Legal Analysis, 2-I, p. 30I-358. 